\title{
Green's tensor technique for scattering in two-dimensional stratified media
}

\author{
Michael Paulus ${ }^{1,2}$ and Olivier J. F. Martin ${ }^{1, *}$ \\ ${ }^{1}$ Electromagnetic Fields and Microwave Electronics Laboratory, Swiss Federal Institute of Technology, ETH-Zentrum ETZ, \\ CH-8092 Zurich, Switzerland \\ ${ }^{2}$ IBM Research, Zurich Research Laboratory, CH-8803 Rüschlikon, Switzerland
}

(Received 1 February 2001; published 29 May 2001)

\begin{abstract}
We present an accurate and self-consistent technique for computing the electromagnetic field in scattering structures formed by bodies embedded in a stratified background and extending infinitely in one direction (two-dimensional geometry). With this fully vectorial approach based on the Green's tensor associated with the background, only the embedded scatterers must be discretized, the entire stratified background being accounted for by the Green's tensor. We first derive the formulas for the computation of this dyadic and discuss in detail its physical substance. The utilization of this technique for the solution of scattering problems in complex structures is then illustrated with examples from photonic integrated circuits (waveguide grating couplers with varying periodicity).
\end{abstract}

DOI: 10.1103/PhysRevE.63.066615

PACS number(s): 42.25.-p, 42.79.Gn, 42.82.Et, 02.60.Cb

\section{INTRODUCTION}

The accurate computation of light scattering from particles in the presence of a stratified background is extremely important for the understanding of realistic structures. Ridges on a multilayered waveguide [1], opaque regions on a contact lithography mask [2], polarization gratings on a transparent backplane [3], and nanowires deposited on a substrate for surface-enhanced Raman scattering [4] all have in common that dielectric or metallic scatterers are distributed in a medium consisting of several layers with different permittivities.

Recently, we presented a technique for computing the propagation and scattering of light in three-dimensional (3D) structures formed by a stratified background with embedded scatterers of finite extension in all three dimensions $[5,6]$. This approach is based on the Green's tensor associated with the stratified background. In this paper, we extend this technique to two-dimensional (2D) geometries, i.e., systems with a translation symmetry in one direction.

A typical 2D system that we want to study is shown in Fig. 1. Several scatterers described by the permittivity $\varepsilon(\mathbf{r})$ are embedded in a stratified background and illuminated with an incident field $\mathbf{E}^{0}$. The stratified background is composed of $L$ layers with relative permittivity $\varepsilon_{l}, l=1, \ldots, L$, and the scatterers extend infinitely along the $y$ axis so that the material system is invariant in that direction. If also the excitation has such a translation symmetry, we can restrict the study of the 3D system (Fig. 1) to a 2D cross section in the $x z$ plane (Fig. 2). We then define the coordinate $\mathbf{r}_{\|}$parallel to this plane,

$$
\mathbf{r}=\left(\mathbf{r}_{\|}, r_{y}\right)=\left(r_{x}, r_{z}, r_{y}\right),
$$

and the parallel wave vector $\mathbf{k}_{\|}$,

$$
\mathbf{k}=\left(\mathbf{k}_{\|}, k_{y}\right)=\left(k_{x}, k_{z}, k_{y}\right) .
$$

\footnotetext{
*Correspondence author. Email address: martin@ifh.ee.ethz.ch
}

Let us emphasize that it is not necessary that also the incident field $\mathbf{E}^{0}$ propagates in the $x z$ plane (Fig. 1). The sole constraint is that $\mathbf{E}^{0}$ has an $\exp \left(i k_{y}^{0} y\right)$ dependence on the symmetry direction $y$. For example, a plane wave

$$
\mathbf{E}^{0}(\mathbf{r}, t)=\mathbf{E}^{0} \exp \left(i \mathbf{k}^{0} \mathbf{r}-i \omega t\right)=\mathbf{E}^{0} \exp \left(i \mathbf{k}_{\|}^{0} \mathbf{r}_{\|}-i \omega t\right) \exp \left(i k_{y}^{0} y\right)
$$

at oblique incidence on the structure fulfills this condition (Figs. 1 and 2).

However, if $\mathbf{E}^{0}$ propagates in the $x z$ plane $\left(k_{y}^{0}=0\right)$, it is possible to decompose the total field into a transverse electric (TE) part with the electric field in the $x z$ plane, and a transverse magnetic (TM) part with the electric field parallel to

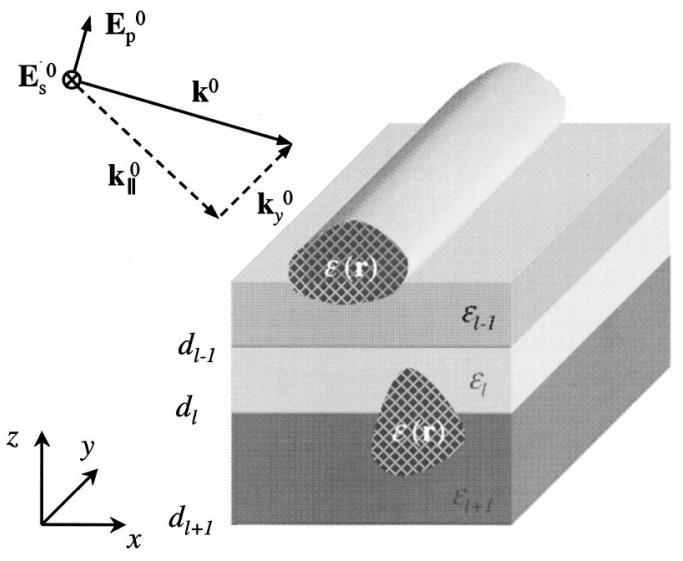

FIG. 1. Schematic view of a 2D scattering system. Several scatterers with permittivity $\varepsilon(\mathbf{r})$ are embedded in a stratified background formed by $L$ layers with permittivity $\varepsilon_{l}, l=1, \ldots, L$. The scatterers are infinitely extended in the $y$ direction. However, the propagation of the incident field is not restricted and its wave vector $\mathbf{k}^{0}=\mathbf{k}_{\|}^{0}+\mathbf{k}_{y}^{0}$ can have components parallel and perpendicular to the $x z$ plane. Similarly, the electric field can be split into two contributions: the $p$-polarized part $\mathbf{E}_{p}^{0}$ lying within the plane of incidence formed by $\mathbf{k}^{0}$ and the $z$ axis, and the $s$-polarized part $\mathbf{E}_{s}^{0}$ standing perpendicularly to this plane. If $\mathbf{k}^{0}=\mathbf{k}_{\|}^{0}\left(\mathbf{k}_{y}^{0}=0\right), p$ polarization is referred to as TE and $s$ polarization as TM. 


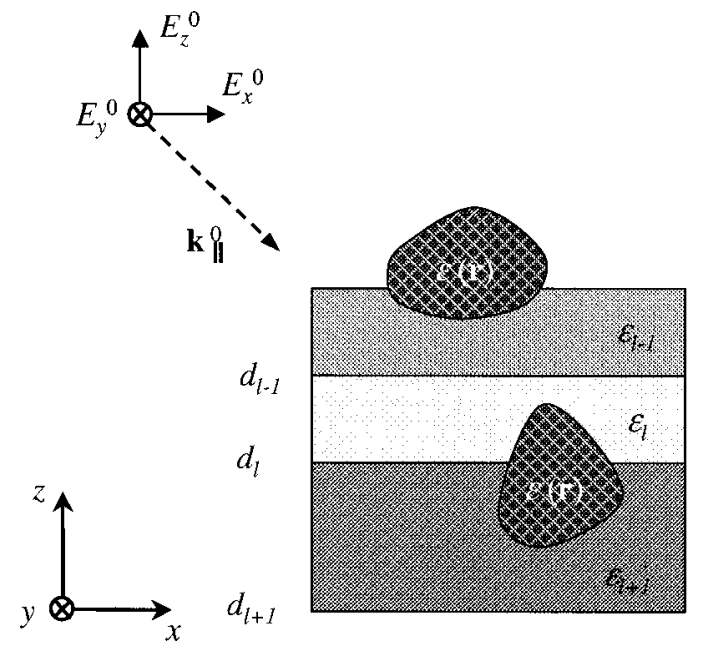

FIG. 2. Projection of Fig. 1 on the $x z$ plane. This plane is normal to the direction of translation symmetry and the investigation of the system can be restricted to this plane. Note that only the projection $\mathbf{k}_{\|}^{0}$ of the incident wave vector $\mathbf{k}^{0}$ is shown and in general all three components of the incident electric field are nonzero.

the $y$ direction. These two polarizations are then decoupled and the scattered field conserves the polarization of the incident field, i.e., the $x$ and $z$ field components are independent of the $y$ component. This is not the case for oblique incidence $\left(k_{y}^{0} \neq 0\right)$ : all three field components are coupled together and new components that were not present in the incident field can be created during the scattering process [7].

For the investigation of such 2D geometries, many useful techniques have already been developed [8-21]. However, most of them strongly depend on the particular geometry under study. The aim of this paper is to present a very general technique for scattering calculations in 2D systems where the scatterers are embedded in a background formed by an arbitrary number of layers. The description of our approach in Sec. II consists of two parts. First, we study the general solution of the scattering problem using the Green's tensor associated with the background of the system. We then derive this Green's tensor and discuss its numerical computation. In Sec. III, we illustrate the technique with two examples and summarize in Sec. IV. All the detailed formulas required for the practical implementation of this method are given in the Appendix.

\section{FORMALISM}

\section{A. Solution of the 2D scattering problem}

To obtain the solution of the 2D scattering problem depicted in Figs. 1 and 2, let us start with the more general solution of the corresponding 3D problem as developed in Refs. $[5,6]$. We consider nonmagnetic materials with relative permeability $\mu=1$ and harmonic fields with time dependence $\exp (-i \omega t)$.

When a system formed by a stratified background with embedded scatterers is illuminated with an incident electric field $\mathbf{E}^{0}(\mathbf{r})$, the total field $\mathbf{E}(\mathbf{r})$ is given by the volume integral equation:

$$
\mathbf{E}(\mathbf{r})=\mathbf{E}^{0}(\mathbf{r})+\int_{V} d \mathbf{r}^{\prime} \mathbf{G}_{3 \mathrm{D}}\left(\mathbf{r}, \mathbf{r}^{\prime}\right) \cdot k_{0}^{2} \Delta \varepsilon\left(\mathbf{r}^{\prime}\right) \mathbf{E}\left(\mathbf{r}^{\prime}\right),
$$

where $\mathbf{G}_{3 \mathrm{D}}\left(\mathbf{r}, \mathbf{r}^{\prime}\right)$ is the Green's tensor associated with the stratified background, $k_{0}^{2}=\omega^{2} \varepsilon_{0} \mu_{0}$ is the vacuum wave number, and $\Delta \varepsilon(\mathbf{r})$ is the dielectric contrast:

$$
\Delta \varepsilon(\mathbf{r})=\varepsilon(\mathbf{r})-\varepsilon_{\kappa}, \quad \mathbf{r} \in \text { layer } \kappa .
$$

The integration in Eq. (4) runs over the scatterer volume $V$. The Green's tensor $\mathbf{G}_{3 \mathrm{D}}\left(\mathbf{r}, \mathbf{r}^{\prime}\right)$ is the solution of the vector wave equation with a point source term [22],

$$
\begin{array}{r}
\boldsymbol{\nabla} \times \boldsymbol{\nabla} \times \mathbf{G}_{3 \mathrm{D}}\left(\mathbf{r}, \mathbf{r}^{\prime}\right)-k_{0}^{2} \varepsilon_{\kappa} \mathbf{G}_{3 \mathrm{D}}\left(\mathbf{r}, \mathbf{r}^{\prime}\right)= \\
\mathbf{1} \delta\left(\mathbf{r}-\mathbf{r}^{\prime}\right), \\
\mathbf{r} \in \text { layer } \kappa,
\end{array}
$$

and represents the electric field in the stratified background radiated at $\mathbf{r}$ by three orthogonal unit dipoles located at $\mathbf{r}^{\prime}$.

In a $2 \mathrm{D}$ system, where the scatterer volume extends infinitely in the $y$ direction, the dielectric contrast does not depend on this coordinate. Since we assume, as discussed in the Introduction, that all the fields have a plane-wave dependence in the $y$ direction, the electric field can be written as

$$
\mathbf{E}(\mathbf{r})=\mathbf{E}\left(\mathbf{r}_{\|}\right) \exp \left(i k_{y}^{0} y\right) .
$$

Let us note that the wave-vector component $k_{y}^{0}$, which is tangential to the different material interfaces, is constant throughout the entire stratified background. It is therefore solely determined by the illumination field and remains conserved in the scattered field. Hence, Eq. (4) can be rewritten as

$$
\mathbf{E}\left(\mathbf{r}_{\|}\right)=\mathbf{E}^{0}\left(\mathbf{r}_{\|}\right)+\int_{A} d \mathbf{r}_{\|}^{\prime} \mathbf{G}_{2 \mathrm{D}}\left(\mathbf{r}_{\|}, \mathbf{r}_{\|}^{\prime}\right) \cdot k_{0}^{2} \Delta \varepsilon\left(\mathbf{r}_{\|}^{\prime}\right) \mathbf{E}\left(\mathbf{r}_{\|}^{\prime}\right)
$$

where we introduced

$$
\mathbf{G}_{2 \mathrm{D}}\left(\mathbf{r}_{\|}, \mathbf{r}_{\|}^{\prime}\right)=\int_{-\infty}^{\infty} d y^{\prime} \mathbf{G}_{3 \mathrm{D}}\left(\mathbf{r}, \mathbf{r}^{\prime}\right) \exp \left[i k_{y}^{0}\left(y^{\prime}-y\right)\right] .
$$

Note that the integration in Eq. (8) runs only over the scatterers section $A$. Recalling that $\mathbf{G}_{3 \mathrm{D}}\left(\mathbf{r}, \mathbf{r}^{\prime}\right)$ corresponds to a point source, $\mathbf{G}_{2 \mathrm{D}}\left(\mathbf{r}_{\|}, \mathbf{r}_{\|}^{\prime}\right)$ gives the field generated by an infinite line source extending in the $y$ direction and represents the 2D Green's tensor associated with the stratified background.

As for 3D systems [6], the Green's tensor can be split into two parts:

$$
\begin{gathered}
\mathbf{G}_{2 \mathrm{D}}\left(\mathbf{r}_{\|}, \mathbf{r}_{\|}^{\prime}\right)=\delta_{\kappa \kappa^{\prime}} \mathbf{G}_{2 \mathrm{D}}^{D}\left(\mathbf{r}_{\|}, \mathbf{r}_{\|}^{\prime}\right)+\mathbf{G}_{2 \mathrm{D}}^{I}\left(\mathbf{r}_{\|}, \mathbf{r}_{\|}^{\prime}\right), \\
\mathbf{r}_{\|} \in \text { layer } \kappa, \mathbf{r}_{\|}^{\prime} \in \text { layer } \kappa^{\prime} .
\end{gathered}
$$

$\mathbf{G}_{2 \mathrm{D}}^{D}$ corresponds to the direct field from $\mathbf{r}_{\|}^{\prime}$ to $\mathbf{r}_{\|}$and is given in closed form by the 2D Green's tensor for an infinite homogeneous material of permittivity $\varepsilon_{\kappa}$ [7]. The indirect part $\mathbf{G}_{2 \mathrm{D}}^{I}$ accounts for all the reflections and refractions at the interfaces and must be evaluated numerically. Since the di- 
vergence of the Green's tensor for $\mathbf{r}_{\|} \rightarrow \mathbf{r}_{\|}^{\prime}$ is completely included in $\mathbf{G}_{2 \mathrm{D}}^{D}$, it can be treated in a similar way to that for an infinite homogeneous medium. Taking the principal value as in Eq. (7) in Ref. [7], we rewrite Eq. (8) as

$$
\begin{aligned}
\mathbf{E}\left(\mathbf{r}_{\|}\right)= & \mathbf{E}^{0}\left(\mathbf{r}_{\|}\right)+\int_{A} d \mathbf{r}_{\|}^{\prime} \mathbf{G}_{2 \mathrm{D}}^{I}\left(\mathbf{r}_{\|}, \mathbf{r}_{\|}^{\prime}\right) \cdot k_{0}^{2} \Delta \varepsilon\left(\mathbf{r}_{\|}^{\prime}\right) \mathbf{E}\left(\mathbf{r}_{\|}^{\prime}\right) \\
& +\lim _{\delta_{A} \rightarrow 0} \int_{A-\delta A} d \mathbf{r}_{\|}^{\prime} \delta_{\kappa \kappa^{\prime}} \mathbf{G}_{2 \mathrm{D}}^{D}\left(\mathbf{r}_{\|}, \mathbf{r}_{\|}^{\prime}\right) \cdot k_{0}^{2} \Delta \varepsilon\left(\mathbf{r}_{\|}^{\prime}\right) \mathbf{E}\left(\mathbf{r}_{\|}^{\prime}\right) \\
& -\mathbf{L} \cdot \frac{\Delta \varepsilon\left(\mathbf{r}_{\|}\right)}{\varepsilon_{\kappa}} \mathbf{E}\left(\mathbf{r}_{\|}\right)
\end{aligned}
$$

where the infinitesimal area $\delta A$ centered at $\mathbf{r}_{\|}$is used to exclude the singularity. The source dyadic $\mathbf{L}$ depends on the shape of $\delta A$ and is given in detail by Yaghjian [23].

\section{B. Discretized 2D equation}

The integral equation (11) is solved numerically on a two dimensional grid with $N$ meshes centered at $\mathbf{r}_{\|, i} \in$ layer $\kappa_{i}$ with area $A_{i}, i=1, \ldots, N$, and dielectric contrast $\Delta \varepsilon_{i}$ $=\Delta \varepsilon\left(\mathbf{r}_{\|, i}\right)$. The discretization need not be regular but can vary locally to enhance the required accuracy, with a smaller mesh where the dielectric contrast $\Delta \varepsilon\left(\mathbf{r}_{\|}\right)$is large. Introducing the discretized field $\mathbf{E}_{i}=\mathbf{E}\left(\mathbf{r}_{\|, i}\right)$ and the discretized Green's tensors $\mathbf{G}_{i, j}^{D}=\mathbf{G}_{2 \mathrm{D}}^{D}\left(\mathbf{r}_{\|, i}, \mathbf{r}_{\|, j}\right)$ and $\mathbf{G}_{i, j}^{I}=\mathbf{G}_{2 \mathrm{D}}^{I}\left(\mathbf{r}_{\|, i}, \mathbf{r}_{\|, j}\right)$, the discretized version of Eq. (11) reads

$$
\begin{gathered}
\mathbf{E}_{i}=\mathbf{E}_{i}^{0}+\sum_{j=1}^{N} \mathbf{G}_{i, j}^{I} \cdot k_{0}^{2} \Delta \varepsilon_{j} \mathbf{E}_{j} A_{j} \\
+\sum_{j=1, j \neq 1} \delta_{\kappa_{i} \kappa_{j}} \mathbf{G}_{i, j}^{D} \cdot k_{0}^{2} \Delta \varepsilon_{j} \mathbf{E}_{j} A_{j} \\
+\mathbf{M}_{i} \cdot k_{0}^{2} \Delta \varepsilon_{i} \mathbf{E}_{i}-\mathbf{L} \cdot \frac{\Delta \varepsilon_{i}}{\varepsilon_{\kappa_{i}}} \mathbf{E}_{i}, \\
i=1, \ldots, N
\end{gathered}
$$

with the self-term $\mathbf{M}_{i}$ defined as

$$
\mathbf{M}_{i}=\lim _{\delta A \rightarrow 0} \int_{A_{i}-\delta A} d \mathbf{r}_{\|}^{\prime} \mathbf{G}_{2 \mathrm{D}}^{D}\left(\mathbf{r}_{\|, i}, \mathbf{r}_{\|}^{\prime}\right) .
$$

Since the tensors $\mathbf{L}$ and $\mathbf{M}_{i}$ come from the direct contribution, we can use their analytic form for a homogeneous polarizable background with permittivity $\varepsilon_{\kappa}$, as given in Ref. [7]. The system of equations (12) is best solved using an iterative solver such as conjugate gradients [24].

\section{2D Green's tensor for a stratified medium}

Equation (9) shows that the Green's tensor for the 2D system can be directly obtained from $\mathbf{G}_{3 \mathrm{D}}\left(\mathbf{r}, \mathbf{r}^{\prime}\right)$ by integration along the translation axis $y$. Whereas $\mathbf{G}_{3 \mathrm{D}}\left(\mathbf{r}, \mathbf{r}^{\prime}\right)$ for an infinite homogeneous background can be expressed analytically [7], this is not possible when the background is stratified. However, a formulation can be found that provides a numerical solution for $\mathbf{G}_{3 \mathrm{D}}\left(\mathbf{r}, \mathbf{r}^{\prime}\right)$ in that case, as explained in detail in Ref. [5]. Let us briefly summarize this procedure:
The Green's tensor is expressed in reciprocal space ( $\mathbf{k}$ space), where the boundary conditions at the stratification interfaces can be enforced more easily. Since we need the Green's tensor in direct space, an inverse Fourier transform is performed and, after analytical integration over $k_{z}(z$ is the stratification direction), we find the following expression for the Green's tensor associated with a stratified background [Eq. (10) in Ref. [5]]:

$$
\begin{aligned}
\mathbf{G}_{3 \mathrm{D}}\left(\mathbf{r}, \mathbf{r}^{\prime}\right)= & -\frac{\hat{\mathbf{z}}}{k_{\kappa}^{2}} \delta(\mathbf{R})+\frac{i}{8 \pi^{2}} \iint d k_{x} d k_{y} \\
& \times \exp \left\{i\left[k_{x}\left(x-x^{\prime}\right)+k_{y}\left(y-y^{\prime}\right)\right]\right\} \\
& \times\left[\mathbf{h}^{s}\left(k_{x}, k_{y} ; z, z^{\prime}\right)+\mathbf{h}^{p}\left(k_{x}, k_{y} ; z, z^{\prime}\right)\right],
\end{aligned}
$$

where $\mathbf{R}=\left(x-x^{\prime}, y-y^{\prime}, z-z^{\prime}\right)$ and $k_{\kappa}^{2}=\omega^{2} \varepsilon_{\kappa} \mu_{\kappa}$ with $\mathbf{r}$ $\in$ layer $\kappa$. The tensors $\mathbf{h}^{s}$ and $\mathbf{h}^{p}$ take into account all the reflections and refractions at the different interfaces. They are determined by the boundary conditions for the electric and the magnetic fields and can be split into two parts corresponding to the $s$ - and $p$-polarized waves, as indicated by the superscript.

Inserting Eq. (14) in Eq. (9), the integral over $y^{\prime}$ can be identified with a $\delta$ function:

$$
\begin{aligned}
\mathbf{G}_{2 \mathrm{D}}\left(\mathbf{r}, \mathbf{r}^{\prime}\right)= & -\frac{\hat{\mathbf{z}} \hat{\mathbf{z}}}{k_{\kappa}^{2}} \delta(\mathbf{R})+\frac{i}{4 \pi} \iint d k_{x} d k_{y} \\
& \times \exp \left[i k_{x}\left(x-x^{\prime}\right)\right] \exp \left[i\left(k_{y}-k_{y}^{0}\right) y\right] \delta\left(k_{y}-k_{y}^{0}\right) \\
& \times\left[\mathbf{h}^{s}\left(k_{x}, k_{y} ; z, z^{\prime}\right)+\mathbf{h}^{p}\left(k_{x}, k_{y} ; z, z^{\prime}\right)\right] \\
= & -\frac{\hat{\mathbf{z}} \hat{\mathbf{z}}}{k_{\kappa}^{2}} \delta(\mathbf{R})+\frac{i}{4 \pi} \int_{-\infty}^{\infty} d k_{x} \exp \left[i k_{x}\left(x-x^{\prime}\right)\right] \\
& \times\left[\mathbf{h}^{s}\left(k_{x}, k_{y}^{0} ; z, z^{\prime}\right)+\mathbf{h}^{p}\left(k_{x}, k_{y}^{0} ; z, z^{\prime}\right)\right]
\end{aligned}
$$

Thus, to obtain the 2D Green's tensor, only a onedimensional integral over the transverse component $k_{x}$ must be calculated numerically. The components of the tensors $\mathbf{h}^{s}$ and $\mathbf{h}^{p}$ can be derived from careful evaluation of Eq. (10) in Ref. [5] and are explicitly given in the Appendix.

The numerical evaluation of the remaining integrals [Eq. (15)] is intricate because it involves several poles and branch cuts corresponding to the different electromagnetic modes that can be excited in the stratified medium. However, the mathematical structure of the equations for the 2D case presented here is similar to that of the 3D case detailed in Ref. [5]. A difference at first view is that in $2 \mathrm{D}$ the integrals run along the entire $k_{x}$ axis while for 3D the integration path is restricted to the semi-infinite positive $k_{\rho}$ axis. However, in 2D the component functions of $\mathbf{h}^{s}$ and $\mathbf{h}^{p}$ are either even or odd symmetrical with respect to the integration variable $k_{x}$. It is therefore possible to reduce the quadrature to the semiinfinite positive $k_{x}$ axis. Hence, exactly the same integration technique as proposed in 3D can be applied [5]: We first use an elliptical deformation of the integration path in the fourth 
quadrant of the complex $k_{x}$ plane to avoid the singularities. As in the 3D case, we then have two possibilities to resume the integration: Either we follow the real $k_{x}$ axis or (for $x$ $\left.\neq x^{\prime}\right)$ we deflect the integration path parallel to the imaginary axis with the correct direction chosen in accordance to the sign of $x-x^{\prime}$. In both cases the integrands converge exponentially because of their dependence on $\exp \left(i k_{\kappa z} \mid z\right.$ $\left.-z^{\prime} \mid\right)=\exp \left\{i\left[k_{\kappa}^{2}-k_{x}^{2}-\left(k_{y}^{0}\right)^{2}\right]^{1 / 2}\left|z-z^{\prime}\right|\right\} \rightarrow \exp \left(-k_{x}\left|z-z^{\prime}\right|\right)$ when $k_{x} \rightarrow \infty$ for the real axis integration [see Eqs. (A1)-(A18)], and on $\exp \left(i k_{x}\left|x-x^{\prime}\right|\right)$ [see Eq. (15)] for the deflected integration path. Hence, a comparison between $\left|z-z^{\prime}\right|$ and $\mid x$ $-x^{\prime} \mid$ gives the path that provides fastest convergence. Let us finally note the basic difference between the 2D and 3D integrals: In 3D, the angular integration of exponential functions in $\mathbf{k}$ space leads to Bessel/Hankel functions in the integrand, whereas in $2 \mathrm{D}$, the bare exponential functions remain.

\section{ILLUSTRATIVE EXAMPLES}

\section{A. The Green's tensor as a dipole field}

To clarify the physical substance of the Green's tensor for stratified media, let us start by illustrating some of its basic properties. We consider a simple three-layer system $\varepsilon_{1}=1$, $\varepsilon_{2}=9, \varepsilon_{3}=1$ with varying thickness $h$ of the high permittivity slab, and we study two components of the Green's tensor, $G_{y z}$ and $G_{z y}$, as a function of the $z$ coordinate of the observation point, $\mathbf{r}_{\|}=(\lambda, z)$. The source point $\mathbf{r}_{\|}^{\prime}$ $=(0,1 \mu \mathrm{m})$ is held fixed in the top layer. The vacuum illumination wavelength is $\lambda=633 \mathrm{~nm}$.

Let us first mention that both components vanish if the incident field does not have a component $k_{y}^{0}$ in the $y$ direction. (In that case, TE and TM waves are decoupled and $G_{y x}=G_{x y}=G_{y z}=G_{z y}=0$.) In our example, we use an illumination with a $[11 \overline{1}]$ orientation, so that $k_{y}^{0}=k_{1} / \sqrt{3}$. Figure 3 shows $G_{y z}$ and $G_{z y}$ when the high permittivity layer extends from $z=0$ to $z=430 \mathrm{~nm}$. Since the Green's tensor represents the electric field radiated at $\mathbf{r}$ by three orthogonal unit dipoles at $\mathbf{r}^{\prime}$, its components must fulfill the respective boundary conditions at the interfaces. Correspondingly, $G_{y z}$ (the $y$ component of a $z$-oriented dipole) is continuous across the interfaces, whereas $G_{z y}$ (the $z$ component of a $y$-oriented dipole) jumps by a factor $\varepsilon_{2} / \varepsilon_{1}=9$ and $\varepsilon_{3} / \varepsilon_{2}=\frac{1}{9}$, respectively.

It is quite surprising that in the lower layer $(z<0)$ both components are identical (Fig. 3). Using the integrands given in the Appendix and the iterative derivation of the amplitude coefficients of Appendix A in Ref. [5], it can be shown that for $\mathbf{r}^{\prime}$ and $\mathbf{r}$ in the two outermost layers 1 and $L$, the components of the Green's tensor are coupled:

$$
\frac{G_{\alpha z}}{k_{1 z}}=\frac{G_{z \alpha}}{k_{L z}}, \quad \alpha=x, y,
$$

where $k_{l z}=\left[k_{l}^{2}-k_{x}^{2}-\left(k_{y}^{0}\right)^{2}\right]^{1 / 2}, l=1, L$. Since in our example $\mathbf{r}^{\prime}$ is located in the top layer and $k_{1 z}=k_{3 z}$, the components coincide for $z<0$ (Fig. 3).

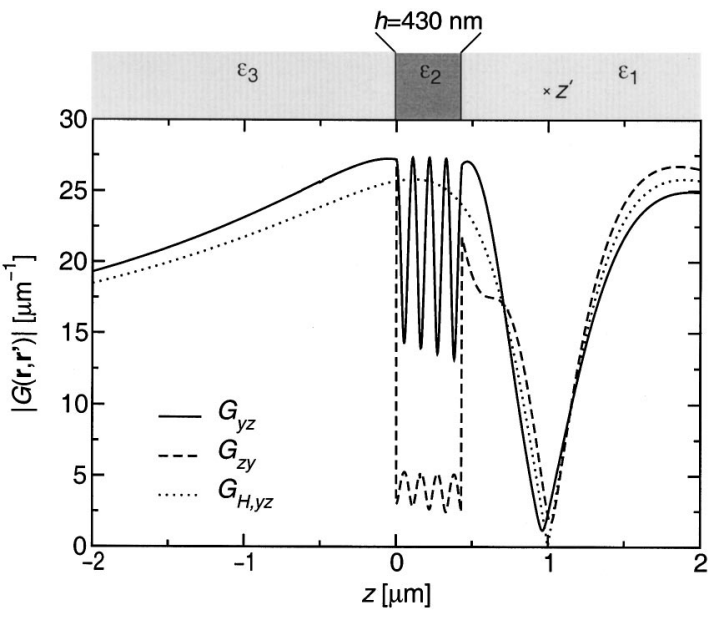

FIG. 3. Green's tensor components for a stratified background $\left(\left|G_{y z}\right|\right.$ and $\left.\left|G_{z y}\right|\right)$ and a homogeneous background $\left(\left|G_{H, y z}\right|\right.$ $\left.=\left|G_{H, z y}\right|\right)$. The stratified structure consists of three layers with $\varepsilon_{1}$ $=1, \varepsilon_{2}=9, \varepsilon_{3}=1$. The high permittivity slab extends from $z=0$ to $z=430 \mathrm{~nm}$ resulting in maximal transmission $\left(\mathcal{T}_{\max }=1\right)$. The permittivity of the homogeneous medium is $\varepsilon_{H}=1$. The line source at $z^{\prime}=1 \mu \mathrm{m}$ is located in the top layer and held fixed. The wavelength is $\lambda=633 \mathrm{~nm}$.

Let us now discuss the relation between the Green's tensor for a stratified medium and that associated with a homogeneous background $\varepsilon_{H}$. When $\mathbf{r}_{\|}-\mathbf{r}_{\|}^{\prime}$ becomes large, the plane-wave expansion of the Green's tensor is mainly governed by the plane wave which propagates in radial direction, $\mathbf{k}_{\|} /\left|\mathbf{k}_{\|}\right|=\left(\mathbf{r}_{\|}-\mathbf{r}_{\|}^{\prime}\right) /\left|\mathbf{r}_{\|}-\mathbf{r}_{\|}^{\prime}\right|$. Hence, with $x$ fixed and $z$ $\rightarrow \pm \infty$, the integrals in Eq. (15) are dominated by the $k_{x}$ $=0$ terms. In this limit, the ratio between the free-space component and the component with stratification is given by the reflection and transmission coefficients of the stratified structure corresponding to this single plane wave. For a three-layer structure, the transmission coefficient $\mathcal{T}$ for $G_{y z}$ and $G_{z y}$ reads [25]

$$
\mathcal{T}=\frac{\mathcal{T}_{12} \mathcal{T}_{23} \exp \left(i h k_{2 z}\right)}{1+\mathcal{R}_{12} \mathcal{R}_{23} \exp \left(2 i h k_{2 z}\right)},
$$

where $\mathcal{R}_{l, l+1}$ and $\mathcal{T}_{l, l+1}$ are the Fresnel reflection and transmission coefficients:

$$
\begin{gathered}
\mathcal{R}_{l, l+1}=\frac{\varepsilon_{l+1} k_{l z}-\varepsilon_{l} k_{l+1 z}}{\varepsilon_{l+1} k_{l z}+\varepsilon_{l} k_{l+1 z}}, \\
\mathcal{T}_{l, l+1}=\frac{2 \varepsilon_{l+1} k_{l z}}{\varepsilon_{l+1} k_{l z}+\varepsilon_{l} k_{l+1 z}} .
\end{gathered}
$$

Note that Eqs. (18a) and (18b) give the coefficients for a $p$-polarized plane wave corresponding to the polarization of $G_{y z}$ and $G_{z y}$ [see Eqs. (A12) and (A16)].

An analysis of Eq. (17) yields a condition for maximal transmission through the slab with thickness $h$ :

$$
h_{\max } k_{2 z}=n \pi,
$$

and for minimal transmission 


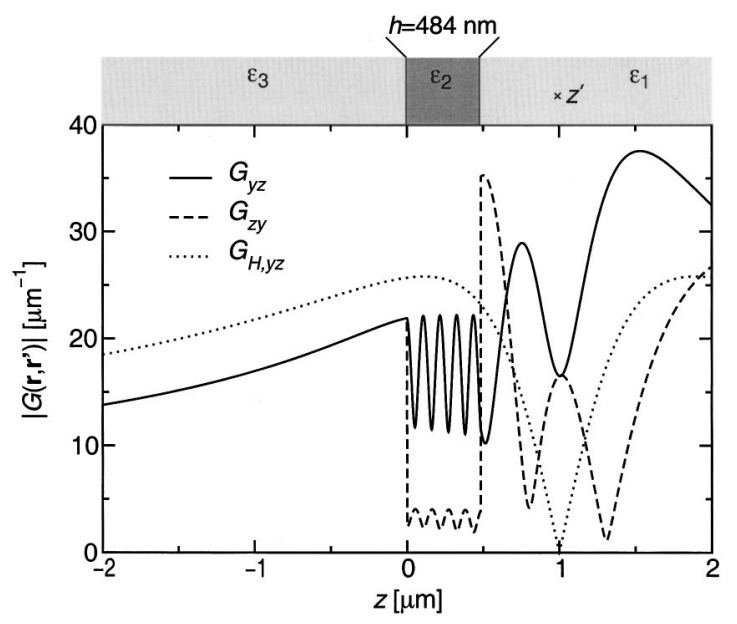

FIG. 4. Same situation as in Fig. 3, but with a high permittivity layer with thickness $h=484 \mathrm{~nm}$. This corresponds to minimal transmission through the $\operatorname{slab}\left(\mathcal{T}_{\min } \approx 0.69\right)$.

$$
h_{\min } k_{2 z}=\left(n+\frac{1}{2}\right) \pi,
$$

where $n=1,2,3, \ldots$ Since for the $k_{y}=0$ terms $k_{2 z}$ $=\sqrt{k_{2}^{2}-\left(k_{y}^{0}\right)^{2}}$, maximal and minimal transmission through the slab of our example are obtained when $h_{\max } \approx n$ $\times 107.5 \mathrm{~nm}$ and $h_{\min } \approx(n+1 / 2) \times 107.5 \mathrm{~nm}$, respectively. The corresponding coefficient of maximal and minimal transmission is $\left|\mathcal{T}_{\max }\right|=1$ and $\left|\mathcal{T}_{\text {min }}\right| \approx 0.69$, respectively [Eq. (17)]. Hence, the plane wave is completely transmitted through a slab with thickness $h_{\max }$ without any reflection.

In Fig. $3(h=430 \mathrm{~nm}=4 \times 107.5 \mathrm{~nm}$, maximal transmission [see Eq. (19a)]), we also report the component $G_{H, y z}=G_{H, z y}$ of the Green's tensor associated with a homogeneous background $\varepsilon_{H}=1$. In the far-field limit $z \rightarrow \pm \infty$, we observe that indeed the components for the stratified structure converge to the free-space solution. At $z$ $= \pm 2 \mu \mathrm{m}$, the difference is less than $5 \%$ and at $z$ $= \pm 10 \mu \mathrm{m}$ it is $\approx 1 \%$ (not shown). For comparison, we also study a second structure with $h=484 \mathrm{~nm} \approx(4+1 / 2)$ $\times 107.5 \mathrm{~nm}$. Figure 4 shows $G_{y z}, G_{z y}$, and $G_{H, y z}$ for this geometry with minimal transmission. At $z=-2 \mu \mathrm{m}$, the ratio $\left|G_{y z} / G_{H, y z}\right| \approx 0.75$ and at $z=-10 \mu \mathrm{m}\left|G_{y z} / G_{H, y z}\right|$ $\approx 0.70$ (not shown) it is close to 0.69 , as expected from the above calculation.

\section{B. Scattering on a planar waveguide grating coupler}

We now use our formalism for a scattering calculation in an integrated optics structure. We consider a silicon-oninsulator (SOI) planar waveguide with a finite rectangular grating on top, as shown in Fig. 5 [26,27]. If a mode propagates in the waveguide and scatters on the grating, light is coupled out of the guiding layer and is transmitted into the air and/or towards the substrate. In Fig. 6, we report the electric-field amplitude $|\mathbf{E}|$ when a grating consisting of 20 protrusions is illuminated with the $\mathrm{TE}_{0}$ mode (transverse electric mode with electric field polarized in the $y$ direction) propagating in the $x$ direction at a wavelength $\lambda=1.3 \mu \mathrm{m}$. The maximum amplitude of the incident mode is normalized

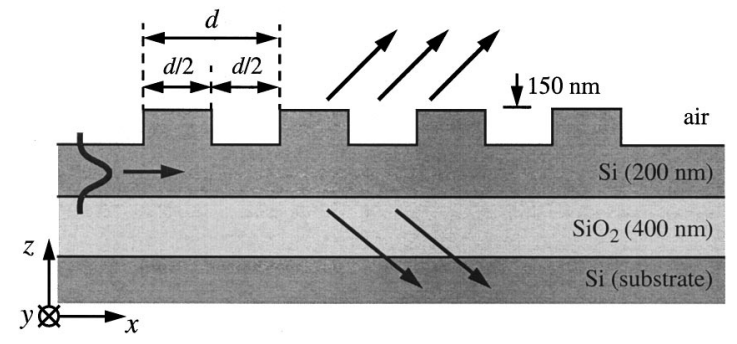

FIG. 5. Geometry of the SOI planar waveguide (permittivities $\varepsilon_{\mathrm{Si}}=12.3, \varepsilon_{\mathrm{SiO}_{2}}=2.1$; wavelength $\lambda=1.3 \mu \mathrm{m}$ ). A Si grating with 150 -nm height, periodicity $d$, and a 0.5 filling factor is etched on top of the waveguide.

to unity. Three periodicities $-d=300 \mathrm{~nm}, d=400 \mathrm{~nm}$, and $d=500 \mathrm{~nm}$-are considered at a fixed filling factor 0.5 . At the left side of all three geometries, a standing wave occurs because of the reflection of the incoming mode by the grating. At the right side, beyond the grating, we can observe an interference pattern created by the interaction of the fields scattered in the forward direction by the different protrusions (and the waveguide mode). The electric-field distributions strongly differ in the three cases. For $d=300 \mathrm{~nm}$, most of the outcoupled light is scattered towards the substrate and almost no light is scattered upwards [Fig. 6(a)]. With increasing periodicity, the main beam of the outcoupled light rotates, so that for $d=400 \mathrm{~nm}$ and $d=500 \mathrm{~nm}$ a larger fraction of the scattered field is transmitted into the air [Figs. 6(b) and 6(c)].

For a more quantitative comparison, we report in Fig. 7 the angular distribution of the electric-field amplitude $|\mathbf{E}|$ far
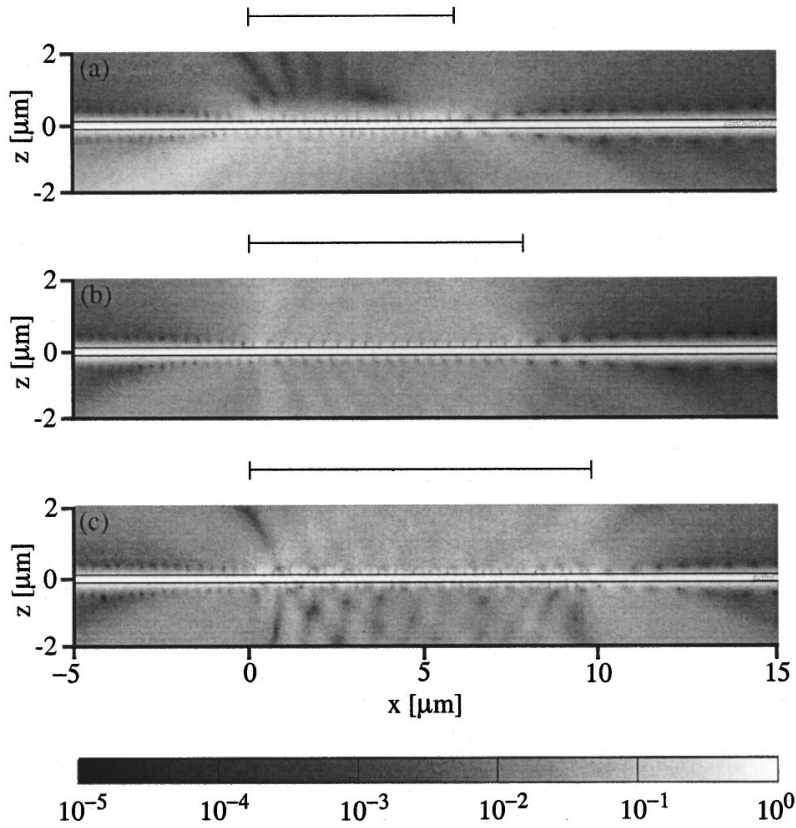

FIG. 6. Electric-field amplitude in the structure of Fig. 5 with three grating periodicities: (a) $d=300 \mathrm{~nm}$, (b) $d=400 \mathrm{~nm}$, and (c) $d=500 \mathrm{~nm}$. The bars represent the total length of each grating. The systems are illuminated with a $\mathrm{TE}_{0}$ mode propagating in the $x$ direction. For clarity, the guiding silicon layer is marked with two lines. A logarithmic color scale is used. 


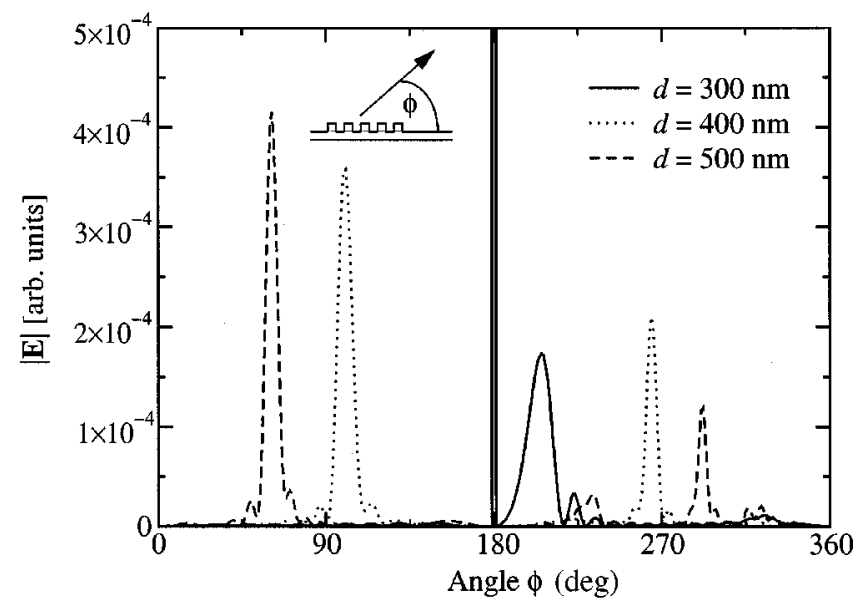

FIG. 7. Angular distribution of the electric-field amplitude $50 \mu \mathrm{m}$ away from the center of the three gratings in Fig. 6. The central peak at $\phi=180^{\circ}$ is caused by the mode in the guiding $\mathrm{Si}$ layer and appears also at $\phi=0^{\circ}$ and $\phi=360^{\circ}$ (not shown).

away from the grating $(50 \mu \mathrm{m})$. Note first that the central peak at $\phi=180^{\circ}$ represents the mode in the guiding layer. Of course, the same peak occurs at $\phi=0^{\circ}$ and $\phi=360^{\circ}$ (not shown). Whereas for $d=300 \mathrm{~nm}$, we can observe a single output beam with an angle $\phi=205^{\circ}$ (backward direction in the substrate), two beams appear for $d=400 \mathrm{~nm}$ and $d$ $=500 \mathrm{~nm}$, respectively: a major one into the air with $\phi$ $=100^{\circ}$ (approximately normal to the waveguide surface) and $\phi=61^{\circ}$ (backward direction in the air), respectively, and a minor one towards the substrate with $\phi=264^{\circ}$ and $\phi$ $=293^{\circ}$, respectively.

In the substrate region one observes for all three periodicities two additional, much broader and smaller peaks at $\phi$ $\approx 225^{\circ}$ and $\phi \approx 325^{\circ}$. A comparison with the field distributions of Fig. 6 shows that these peaks correspond to edge effects at the beginning and at the end of the grating. At these interfaces between the planar geometry and the grating, the mode scatters strongly.

Figure 6 also illustrates that the boundary conditions both at the edges of the computation window and at the different material interfaces are perfectly fulfilled. These boundary conditions are already included in the Green's tensor so that there is no special treatment necessary to avoid unphysical reflections. Let us finally emphasize that the discretization of the geometry is restricted to the protrusions forming the grating. The optical processes in the stratified planar waveguide are completely accounted for in the Green's tensor associated with the background.

\section{SUMMARY}

In this paper, we have presented a Green's tensor approach for the computation of scattering problems with $2 \mathrm{D}$ bodies embedded in a stratified background. We have first described in detail the numerical procedure required for the computation of the Green's tensor associated with a stratified background. We have then demonstrated the utilization of this dyadic to perform scattering calculations in complex 2D systems. From a practical point of view, in addition to truly two-dimensional geometries, 3D structures can often be considered as $2 \mathrm{D}$ when the extension of the scatterers in one particular direction is very large and the electromagnetic field is so well localized that edge effects in that direction become negligible. It is then justified to assume that the system extends to infinity in that particular direction.

\section{ACKNOWLEDGMENTS}

It is a pleasure to acknowledge stimulating discussions with P. Gay-Balmaz, J. P. Kottmann, and E. Moreno and to thank B. Michel (IBM) and R. Vahldieck (ETH) for their support of the project. We gratefully acknowledge the funding from the Swiss National Science Foundation.

\section{APPENDIX}

In this appendix, we explicitly give the integrands of Eq. (15). To impose the boundary conditions at the different interfaces, the components of the tensors $\mathbf{h}^{s}$ and $\mathbf{h}^{p}$ are expressed in terms of upgoing and downgoing $s$-and $p$-polarized plane waves:

$$
h_{x x}^{s}\left(k_{x}\right)=\frac{k_{y}^{0^{2}}}{k_{\kappa z} k_{\rho}^{2}}\left[A_{\kappa}^{s} \exp \left(i k_{\kappa z} z\right)+B_{\kappa}^{s} \exp \left(-i k_{\kappa z} z\right)\right]
$$

$h_{x x}^{p}\left(k_{x}\right)= \pm \frac{k_{x}^{2} k_{\kappa z}}{k_{\kappa}^{2} k_{\rho}^{2}}\left[A_{\kappa, x x}^{p} \exp \left(i k_{\kappa z} z\right)-B_{\kappa, x x}^{p} \exp \left(-i k_{\kappa z} z\right)\right]$

$$
h_{x y}^{s}\left(k_{x}\right)=-\frac{k_{y}^{0} k_{x}}{k_{\kappa z} k_{\rho}^{2}}\left[A_{\kappa}^{s} \exp \left(i k_{\kappa z} z\right)+B_{\kappa}^{s} \exp \left(-i k_{\kappa z} z\right)\right],
$$

$$
h_{x y}^{p}\left(k_{x}\right)= \pm \frac{k_{y}^{0} k_{x} k_{\kappa z}}{k_{\kappa}^{2} k_{\rho}^{2}}\left[A_{\kappa, x y}^{p} \exp \left(i k_{\kappa z} z\right)-B_{\kappa, x y}^{p} \exp \left(-i k_{\kappa z} z\right)\right]
$$

$$
h_{x z}^{s}\left(k_{x}\right)=0
$$

$$
h_{x z}^{p}\left(k_{x}\right)=-\frac{k_{x}}{k_{\kappa}^{2}}\left[A_{\kappa, x z}^{p} \exp \left(i k_{\kappa z} z\right)-B_{\kappa, x z}^{p} \exp \left(-i k_{\kappa z} z\right)\right],
$$$$
h_{y x}^{s}\left(k_{x}\right)=h_{x y}^{s},
$$

$$
h_{y x}^{p}\left(k_{x}\right)=h_{x y}^{p}
$$

$$
h_{y y}^{s}\left(k_{x}\right)=\frac{k_{x}^{2}}{k_{\kappa z} k_{\rho}^{2}}\left[A_{\kappa}^{s} \exp \left(i k_{\kappa z} z\right)+B_{\kappa}^{s} \exp \left(-i k_{\kappa z} z\right)\right]
$$

$$
h_{y y}^{p}\left(k_{x}\right)= \pm \frac{k_{y}^{0^{2}} k_{\kappa z}}{k_{\kappa}^{2} k_{\rho}^{2}}\left[A_{\kappa, y y}^{p} \exp \left(i k_{\kappa z} z\right)-B_{\kappa, y y}^{p} \exp \left(-i k_{\kappa z} z\right)\right],
$$




$$
\begin{gathered}
h_{y z}^{s}\left(k_{x}\right)=0, \\
h_{y z}^{p}\left(k_{x}\right)=-\frac{k_{y}^{0}}{k_{\kappa}^{2}}\left[A_{\kappa, y z}^{p} \exp \left(i k_{\kappa z} z\right)-B_{\kappa, y z}^{p} \exp \left(-i k_{\kappa z} z\right)\right] \\
h_{z x}^{s}\left(k_{x}\right)=0, \\
h_{z x}^{p}\left(k_{x}\right)=\mp \frac{k_{x}}{k_{\kappa}^{2}}\left[A_{\kappa, z x}^{p} \exp \left(i k_{\kappa z} z\right)+B_{\kappa, z x}^{p} \exp \left(-i k_{\kappa z} z\right)\right], \\
h_{z y}^{p}\left(k_{x}\right)=\mp \frac{k_{y}^{0}}{k_{\kappa}^{2}}\left[A_{\kappa, z y}^{p} \exp \left(i k_{\kappa z} z\right)+B_{\kappa, z y}^{p} \exp \left(-i k_{\kappa z} z\right)\right], \\
h_{z z}^{p}\left(k_{x}\right)=\frac{\mathrm{A} 1}{k_{\kappa}^{2} k_{\kappa z}}\left[A_{\kappa, z z}^{p} \exp \left(i k_{\kappa z} z\right)+B_{\kappa, z z}^{p} \exp \left(-i k_{\kappa z} z\right)\right],
\end{gathered}
$$

where $k_{\rho}^{2}=k_{x}^{2}+\left(k_{y}^{0}\right)^{2} \quad$ and $\quad k_{\kappa z}=\left(k_{\kappa}^{2}-k_{\rho}^{2}\right)^{1 / 2}$ with $\mathbf{r}$ $\in$ layer $\kappa$. The upper sign in these equations refers to $z$ $>z^{\prime}$ and the lower sign to $z<z^{\prime}$. The amplitude coefficients $A_{\kappa}^{s}, B_{\kappa}^{s}, A_{\kappa, \alpha \beta}^{p}$, and $B_{\kappa, \alpha \beta}^{p}$ must be calculated for the layer $\kappa$ where $\mathbf{r}$ is located and are functions of $\left(k_{\kappa z} ; z, z^{\prime}\right)$. They are connected to the amplitudes in all other layers and can be found via an iterative scheme completely identical to that of the 3D case given in Appendix A of Ref. [5]. Note that $k_{\kappa z}$ is now only a function of $k_{x}$, since $k_{y}$ is replaced by the constant parameter $k_{y}^{0}$ given by the incident field. Since all non- vanishing amplitude coefficients of $s$-polarized waves are equal, we can suppress the indices $\alpha$ and $\beta$ and simply write $A_{\kappa}^{s}$ and $B_{\kappa}^{s}$.

Let us now consider the special case in which the incident field propagates in the $x z$ plane. With $k_{y}^{0}=0$, the tensors $\mathbf{h}^{s}$ and $\mathbf{h}^{p}$ reduce to

$$
\begin{gathered}
h_{x x}^{p}\left(k_{x}\right)= \pm \frac{k_{\kappa z}}{k_{\kappa}^{2}}\left[A_{\kappa, x x}^{p} \exp \left(i k_{\kappa z} z\right)-B_{\kappa, x x}^{p} \exp \left(-i k_{\kappa z} z\right)\right] \\
h_{x z}^{p}\left(k_{x}\right)=-\frac{k_{x}}{k_{\kappa}^{2}}\left[A_{\kappa, x z}^{p} \exp \left(i k_{\kappa z} z\right)-B_{\kappa, x z}^{p} \exp \left(-i k_{\kappa z} z\right)\right] \\
h_{y y}^{s}\left(k_{x}\right)=\frac{1}{k_{\kappa z}}\left[A_{\kappa}^{s} \exp \left(i k_{\kappa z} z\right)+B_{\kappa}^{s} \exp \left(-i k_{\kappa z} z\right)\right], \\
h_{z x}^{p}\left(k_{x}\right)=\mp \frac{k_{x}}{k_{\kappa}^{2}}\left[A_{\kappa, z x}^{p} \exp \left(i k_{\kappa z} z\right)+B_{\kappa, z x}^{p} \exp \left(-i k_{\kappa z} z\right)\right] \\
h_{z z}^{p}\left(k_{x}\right)=\frac{k_{x}^{2}}{k_{\kappa}^{2} k_{\kappa z}}\left[A_{\kappa, z z}^{p} \exp \left(i k_{\kappa z} z\right)+B_{\kappa, z z}^{p} \exp \left(-i k_{\kappa z} z\right)\right],
\end{gathered}
$$

and all other components vanish.

These equations clearly show the decoupling of the two polarizations that can be excited in that case. For the component of the illuminating electric field parallel to the translation symmetry axis $y$ (TM polarization), $\mathbf{G}_{2 \mathrm{D}}$ reduces to a scalar [Eq. $\left.\left(\mathrm{A} 9^{\prime}\right)\right]$. For the components perpendicular to that axis, i.e., lying within the $x z$ plane (TE polarization), $\mathbf{G}_{2 \mathrm{D}}$ reduces to a $(2 \times 2)$ matrix [Eqs. $\left(\mathrm{A} 2^{\prime}\right),\left(\mathrm{A} 6^{\prime}\right),\left(\mathrm{A} 14^{\prime}\right)$, and $\left.\left(\mathrm{A} 18^{\prime}\right)\right]$.
[1] R. März, Integrated Optics Design and Modeling (Artech House, Boston, 1994).

[2] H. Schmid, H. Biebuyck, B. Michel, and O. J. F. Martin, Appl. Phys. Lett. 72, 2379 (1998).

[3] B. Schnabel, E. B. Kley, and F. Wyrowski, Opt. Eng. 38, 220 (1999).

[4] M. Kahl and E. Voges, Phys. Rev. B 61, 14078 (2000).

[5] M. Paulus, P. Gay-Balmaz, and O. J. F. Martin, Phys. Rev. E 62, 5797 (2000).

[6] M. Paulus and O. J. F. Martin, J. Opt. Soc. Am. A 18, 854 (2001).

[7] O. J. F. Martin and N. B. Piller, Phys. Rev. E 58, 3909 (1998).

[8] G. R. Hadley, J. Lightwave Technol. 16, 134 (1998).

[9] H. El-Refaei, D. Yevick, and I. Betty, IEEE Photonics Technol. Lett. 12, 389 (2000).

[10] C. M. Herzinger, C. C. Lu, T. A. DeTemple, and W. C. Chew, IEEE J. Quantum Electron. 29, 2273 (1993).

[11] J. Willems, J. Haes, and R. Baets, Opt. Quantum Electron. 27, 995 (1995).
[12] H. Derudder, D. De Zutter, and F. Olyslager, Electron. Lett. 34, 2138 (1998).

[13] G. Videen and D. Ngo, J. Opt. Soc. Am. A 14, 70 (1997).

[14] G. R. Hadley, J. Lightwave Technol. 16, 142 (1998).

[15] A. Taflove and S. C. Hagness, Computational Electrodynamics: The Finite difference Time domain Method, 2nd ed. (Artech House, Boston, 2000).

[16] S. F. Helfert and R. Pregla, Opt. Quantum Electron. 31, 721 (1999).

[17] W. Huang and R. R. A. Syms, J. Lightwave Technol. 17, 2658 (1999).

[18] D. R. Beltrami, J. D. Love, and F. Ladouceur, Opt. Quantum Electron. 31, 307 (1999).

[19] J. B. Pendry and P. M. Bell, in Photonic Band Gap Materials, Vol. 315 of NATO Advanced Study Institute Series, edited by C. M. Soukoulis (Kluwer, Dordrecht, 1996), pp. 203-228.

[20] P. J. Valle, F. Moreno, and J. M. Saiz, J. Opt. Soc. Am. A 15, 158 (1998).

[21] B. Hu and W. C. Chew, Radio Sci. 35, 31 (2000). 
[22] P. M. Morse and H. Feshbach, Methods of Theoretical Physics (McGraw-Hill, New York, 1953).

[23] A.D. Yaghjian, Proc. IEEE 68, 248 (1980).

[24] P. J. Flatau, Opt. Lett. 22, 1205 (1997).
[25] M. Born and E. Wolf, Principles of Optics, 6th ed. (Pergamon Press, Oxford, 1987).

[26] T. W. Ang et al., IEEE Photonics Technol. Lett. 12, 59 (2000).

[27] R. Orobtchouk et al., Appl. Opt. 39, 5773 (2000). 